\title{
Four-quark condensates and chiral symmetry restoration in a resonance gas model
}

\author{
Stefan Leupold \\ Institut für Theoretische Physik, Universität Giessen, Germany
}

\begin{abstract}
As an alternative to the two-quark condensate we propose a specific four-quark condensate as an order parameter of chiral symmetry restoration. We show that this four-quark condensate is closer connected to observable quantities. Within a resonance gas model we calculate the in-medium changes of two- and four-quark condensate as functions of temperature and baryo-chemical potential. In this way we estimate the line of chiral symmetry restoration in the temperature-potential plane and also as a function of energy and baryon density. It turns out that the line determined from the vanishing of the four-quark condensate is extremely constant as a function of the energy density.
\end{abstract}

\section{INTRODUCTION}

In QCD, the theory of the strong interaction, the masses of the lightest quarks are very light 1] as compared to typical hadronic scales. Therefore, QCD has an approximate chiral symmetry. From the absence of degenerate chiral partners and from the existence of very light pseudoscalar mesons one concludes that chiral symmetry is realized in the Nambu-Goldstone phase, i.e. it is spontaneously broken (see e.g. 2] and references therein). Consequently, the light pseudoscalar states are interpreted as Goldstone bosons.

It is expected that in a hot and dense strongly interacting medium chiral symmetry gets restored. To quantify the degree of restoration of a symmetry one typically uses an order parameter. Indeed, concerning chiral symmetry several quantities are conceivable as an order parameter. There are at least two considerations which decide how useful a possible order parameter actually might be, namely a) observability and b) simple connection to QCD. Clearly, it would be preferable to have a quantity which was observable in vacuum and also in a strongly interacting medium. In this way one could experimentally test the process of chiral symmetry restoration. Unfortunately such a "smoking gun" is hard to find (cf. e.g. 3] ). But it should be clear that it is appreciable as a first step, if the order parameter is at least measurable in the vacuum. From the theoretical point of view it would be preferable to have a quantity which fulfills criterion b), i.e. which can be easily expressed in terms of quark and gluon fields and which can be determined in lattice QCD 4] or quark model calculations. Basically this criterion b) boils down to the requirement that the order parameter is a condensate. It is the purpose of the present paper to propose a specific four-quark condensate as a very useful order parameter. First, however, we shall discuss order parameters which are more frequently considered. We will especially comment how well criteria a) and b) are met.

Obviously, the appearance of Goldstone bosons is closely connected to spontaneous symmetry breaking. Therefore, it is natural that one can find an order parameter of chiral symmetry restoration which is connected to the properties of Goldstone bosons. Indeed, the pion decay constant $F_{\pi}$ is such a quantity. It vanishes, if chiral symmetry is restored. Clearly, $F_{\pi}$ can be extracted experimentally from the life time of the pion. In principle, $F_{\pi}$ is connected to a quark current, but the expression is non-local. In addition, it is not so easy to determine it from lattice QCD (cf. e.g. 5 and references therein). Thus, criterion a) is met by the pion decay constant, but criterion b) only in part.

Frequently used order parameters are the two-quark condensates $\langle\bar{u} u\rangle,\langle\bar{d} d\rangle$ and $\langle\bar{s} s\rangle$ or the corresponding two- or three-flavor averages. ${ }^{1}$ In lattice QCD calculations a dramatic rise of the pressure as a function of the temperature has been observed at a specific temperature $T_{c}$. This points towards a phase transition or at least (and more likely) a rapid crossover. It has been found that the two-quark condensate also shows a drastic change at the very same temperature $T_{c}$ (more precisely, the corresponding susceptibility peaks at this temperature) 6]. This suggests that the two-quark condensate is a proper tool to study chiral symmetry restoration. On the other hand, it might be that the situation is different for non-vanishing baryo-chemical potential, i.e. at finite baryon density. Indeed, one can imagine scenarios where the two-quark condensate vanishes while chiral symmetry is still broken (see e.g. 7]). On top of that problem, criterion a) is not met by the two-quark condensate: It is not directly connected to an observable quantity. Here a closer look is useful: In the standard scenario of chiral symmetry breaking ( $\chi \mathrm{SB})$ one gets [8] (see also $[9]$ )

$$
F_{\pi}^{2} M_{\pi}^{2} \approx-\underbrace{\left(m_{u}+m_{d}\right)}_{=: 2 m_{q}}\langle\bar{u} u\rangle \approx-\left(m_{u}+m_{d}\right)\langle\bar{d} d\rangle
$$

\footnotetext{
${ }^{1}$ For the following qualitative discussion we will simply talk about the two-quark condensate without specifying the flavor. When it comes to quantitative statements we will specify which two-quark condensate is considered.
} 
(where isospin-breaking effects are neglected for the condensates). We see that it is not simply the two-quark condensate but its combination with the quark mass which is connected to observables ${ }^{2}$ - and also here not to one observable, but to a combination of two of them, the pion decay constant and the pion mass $M_{\pi}$. From (1) it is obvious that it is possible that the two-quark condensate vanishes with a pion decay constant which stays more or less constant. A change of the pion mass would be sufficient to change the condensate. In such a case, chiral symmetry would still be broken. Besides (11) there is so far no relation derived from first principles which directly connects two-quark condensates with observable quantities.

To summarize, the pion decay constant satisfies criterion a), but no so much b), whereas it is just the other way round for the two-quark condensate. Therefore, it is reasonable to explore also other order parameters of chiral symmetry breaking. Note that not much lattice QCD studies have been performed here. The mixed quark-gluon condensate has been studied on the lattice in [11]. We will briefly comment on this condensate below (section IV]. In the present paper we will mainly concentrate on a specific four-quark condensate. In the next section we will motivate in detail why this condensate qualifies as a useful order parameter. So far there are no lattice QCD calculations available for the temperature dependence of this four-quark condensate. In addition, an extension of lattice QCD to finite baryo-chemical potentials is still complicated (cf. e.g. [12, 13]). Therefore, we will evaluate the in-medium expectation value of the four- and also of the two-quark condensate within a resonance gas approximation in sections III and IV The numerical results are presented in section V A summary and an outlook are provided in section VI In an appendix some formal issues are discussed.

\section{GENERALIZED WEINBERG SUM RULES}

The two main arguments which point towards spontaneous breaking of chiral symmetry are the appearance of light states (interpreted as Goldstone bosons) and the absence of degenerate chiral partners. In the following we focus on the second aspect.

We start with the retarded $(R)$ current-current correlator 14$]$

$$
\Pi_{\mu \nu}(q)=i \int d^{4} x e^{i q x}\left\langle R j_{\mu}^{V}(x) j_{\nu}^{V}(0)\right\rangle
$$

where $j_{\mu}^{V}$ is the electromagnetic current with the quantum numbers of the $\rho$-meson,

$$
j_{\mu}^{V}=\frac{1}{2}\left(\bar{u} \gamma_{\mu} u-\bar{d} \gamma_{\mu} d\right)
$$

The expectation value for an arbitrary operator $\mathcal{O}$ is defined by

$$
\langle\mathcal{O}\rangle:=\frac{\operatorname{Tr}\left(\mathcal{O} e^{-\beta(H-\mu N)}\right)}{\operatorname{Tr}\left(e^{-\beta(H-\mu N)}\right)}
$$

with the Hamiltonian $H$, the baryon number operator $N$, the inverse temperature $\beta=1 / T$, the baryo-chemical potential $\mu$ and the trace $\mathrm{Tr}$ is taken over all possible $n$-body states.

In a first step, we will decompose the Lorentz tensor $\Pi_{\mu \nu}(q)$. In the vacuum we only had the structures $g_{\mu \nu}$ and $q_{\mu} q_{\nu}$ at hand. An equilibrated medium with finite temperature and baryo-chemical potential introduces an additional Lorentz vector $n_{\mu}$ which is conveniently chosen to be proportional to the (conserved) baryonic current and normalized to 1 . In the rest system of the medium it is simply given by $n_{\mu}=(1,0,0,0)$. Since the current (3) is conserved by the QCD equations of motion we have

$$
q^{\mu} \Pi_{\mu \nu}(q)=0
$$

We can construct two independent projectors $L_{\mu \nu}(q)$ and $T_{\mu \nu}(q)$ which both still satisfy current conservation $q^{\mu} L_{\mu \nu}(q)=q^{\mu} T_{\mu \nu}(q)=0$ (cf. e.g. [15]):

$$
\begin{aligned}
& L_{\mu \nu}(q)=\frac{\left((q \cdot n) q_{\mu}-q^{2} n_{\mu}\right)\left((q \cdot n) q_{\nu}-q^{2} n_{\nu}\right)}{q^{2}\left(q^{2}-(q \cdot n)^{2}\right)} \\
& T_{\mu \nu}(q)=g_{\mu \nu}-\frac{q_{\mu} q_{\nu}}{q^{2}}-L_{\mu \nu}(q)
\end{aligned}
$$

\footnotetext{
${ }^{2}$ Indeed, only this combination is renormalization group invariant [10].
} 
Hence, the correlator can be decomposed in the following way:

$$
\Pi_{\mu \nu}=\Pi^{T} T_{\mu \nu}+\Pi^{L} L_{\mu \nu} .
$$

The scalar functions $\Pi^{T}$ and $\Pi^{L}$ in general depend on $q^{2}$ and $(q \cdot n)^{2}$. In the rest frame of the medium one can equally well state that $\Pi^{T / L}$ depends on $q^{2}$ and $\vec{q}^{2}=(q \cdot n)^{2}-q^{2}$ with the three-momentum $\vec{q}$ relative to the medium. The projectors $T_{\mu \nu}$ and $L_{\mu \nu}$ introduced in (6) are chosen such that they project on states which are transverse and longitudinal, respectively, with respect to $\vec{q}$.

For simplicity we restrict ourselves in the following to the case of vanishing $\vec{q}$, i.e. where the current is at rest relative to the medium. In this case there is no distinction between longitudinal and transverse states, i.e. $\Pi^{T}$ and $\Pi^{L}$ become equal:

$$
\Pi^{T}\left(q^{2}, \vec{q}^{2}=0\right)=\Pi^{L}\left(q^{2}, \vec{q}^{2}=0\right)=:-q^{2} R^{V}\left(q^{2}\right) .
$$

$R^{V}$ has a direct physical meaning in the time-like region $s=q^{2}>0$. It is related to the cross section $e^{+} e^{-} \rightarrow$ hadrons with isospin 1 via 10 ]

$$
\frac{\sigma^{I=1}\left(e^{+} e^{-} \rightarrow \text { hadrons }\right)}{\sigma\left(e^{+} e^{-} \rightarrow \mu^{+} \mu^{-}\right)}=12 \pi \operatorname{Im} R^{V}
$$

In addition, it is related to the decay of the $\tau$ into a neutrino and an even number of pions [16, 17].

If chiral symmetry is manifestly realized, i.e. not spontaneously broken, the current-current correlator for the vector current (3) and the corresponding one for the axial-vector current

$$
j_{\mu}^{A}=\frac{1}{2}\left(\bar{u} \gamma_{\mu} \gamma_{5} u-\bar{d} \gamma_{\mu} \gamma_{5} d\right)
$$

must be degenerate. In other words, in this case the spectrum of hadronic states which couples to $j^{V}$ must be degenerate with the corresponding spectrum which couples to $j^{A}$. These are the mentioned chiral partners. If chiral symmetry is spontaneously broken, there are no degenerate chiral partners and the following Weinberg sum rules [18] hold (in the chiral limit)

$$
\begin{gathered}
\frac{1}{\pi} \int_{0}^{\infty} d s\left(\operatorname{Im} R^{V}(s)-\operatorname{Im} R^{A}(s)\right)=F_{\pi}^{2}, \\
\frac{1}{\pi} \int_{0}^{\infty} d s s\left(\operatorname{Im} R^{V}(s)-\operatorname{Im} R^{A}(s)\right)=0 .
\end{gathered}
$$

The quantity $R^{V}$ is connected to the vector correlator according to 8 . $R^{A}$ is the corresponding quantity for the axial-vector current (10) with one subtle but important difference: In principle, also the pion contributes to $R^{A}$ with a strength determined by the pion decay constant $F_{\pi}$. This contribution is taken out from $R^{A}$ and displayed explicitly in (11a). Actually both quantities $R^{V}$ and $R^{A}$ can be observed in $\tau$ decays, if one triggers on an even (for $R^{V}$ ) or odd (for $R^{A}$ ) number of pions in the final state [16, 17].

The sum rules (11) have been derived prior to the advent of QCD by using current algebra [18]. In other words, they are independent of QCD. In principle, one could also weight the difference $R^{V}-R^{A}$ with higher powers of $s$. In contrast to (11) such integrals are specific to QCD since quark fields and the strong coupling are involved (see below).

From a practical point of view the predictions (11) cannot be tested as they stand, since the integrals cover the whole range up to infinity. On the other hand, we expect that for large $s$ the inclusive cross section in (9) and therefore $R^{V}$ should be given by perturbative QCD $[19]$. Since perturbative QCD does not show chiral symmetry breaking we expect the same behavior for the axial-vector correlator, i.e. [19, 20]

$$
\operatorname{Im} R^{A}(s)=\operatorname{Im} R^{V}(s)=\frac{1}{8 \pi}\left(1+\frac{\alpha_{s}}{\pi}\right) \quad \text { for } \quad s>s_{0}
$$

with a threshold value $s_{0}$. 
Using (12) we can restrict the integration limits in (11) and get

$$
\begin{aligned}
\frac{1}{\pi} \int_{0}^{s_{0}} d s\left(\operatorname{Im} R^{V}(s)-\operatorname{Im} R^{A}(s)\right) & =F_{\pi}^{2}, \\
\frac{1}{\pi} \int_{0}^{s_{0}} d s s\left(\operatorname{Im} R^{V}(s)-\operatorname{Im} R^{A}(s)\right) & =0, \\
\frac{1}{\pi} \int_{0}^{s_{0}} d s s^{2}\left(\operatorname{Im} R^{V}(s)-\operatorname{Im} R^{A}(s)\right) & =-\frac{1}{2} \pi \alpha_{s}\left\langle\mathcal{O}_{\chi \mathrm{SB}}\right\rangle .
\end{aligned}
$$

We have supplemented the Weinberg sum rules by a third sum rule (13c) which — as already announced — is specific for QCD 20]. It involves the four-quark condensate

$$
\left\langle\mathcal{O}_{\chi \mathrm{SB}}\right\rangle=\left\langle\left(\bar{u} \gamma_{\mu} \gamma_{5} \lambda^{a} u-\bar{d} \gamma_{\mu} \gamma_{5} \lambda^{a} d\right)^{2}-\left(\bar{u} \gamma_{\mu} \lambda^{a} u-\bar{d} \gamma_{\mu} \lambda^{a} d\right)^{2}\right\rangle
$$

where $\lambda_{a}$ denotes the color Gell-Mann matrices. For an in-medium generalization of (13) see also 21].

Actually the quantity $\alpha_{s}\left\langle\mathcal{O}_{\chi \mathrm{SB}}\right\rangle$ is not a renormalization group invariant object [22]. A serious derivation of (13c) reveals that it must be evaluated at the scale $s_{0}$ (cf. 23] and references therein). There are also radiative corrections which we have not displayed explicitly. Since the time when four-quark condensates were introduced 20 it is a lively discussed issue whether they can be related to the two-quark condensate by factorization (see e.g. 24] and references therein). This discussion is complicated by the fact that four-quark condensates are difficult to determine experimentally. In addition, also the size of the two-quark condensate is not so well determined: With (11) one can relate the two-quark condensate to the very well known pion mass and decay constant and to the much less known current quark masses 1]. This uncertainty doubles since under the assumption of factorization the square of the two-quark condensate is connected to the four-quark condensates. With all these problems in mind we note that it has been found recently in [25] that in vacuum the four-quark condensate (14) indeed factorizes. This finding is based on the recent data on $\tau$ decays and on a technique which we will describe in a moment. On the theory side there is one particular limit in which vacuum four-quark condensates are connected to the two-quark condensate, namely for a large number of colors $N_{c}[26$. For in-medium condensates this is again more subtle [24]. We will come back to that point below.

From a phenomenological point of view the sum rules (13) are still unsatisfying: As already mentioned, nowadays experiments can address the difference of correlators in $\tau$ decays. Therefore, one can explore the integrals of (13) only up to energies below the $\tau$ mass. It turns out that the left hand sides of the sum rules are not convergent as a function of the threshold $s_{0}$ for values below the $\tau$ mass (squared). By proper combinations of (13) one can, however, decrease the sensitivity on $s_{0}$ and obtain sum rules which show a much better convergence behavior already at the experimentally accessible energies [23, 25]:

$$
\begin{aligned}
& \frac{1}{\pi s_{0}} \int_{0}^{s_{0}} d s\left(s_{0}-s\right)\left(\operatorname{Im} R^{V}(s)-\operatorname{Im} R^{A}(s)\right)=F_{\pi}^{2}, \\
& \frac{1}{\pi} \int_{0}^{s_{0}} d s s\left(s_{0}-s\right)\left(\operatorname{Im} R^{V}(s)-\operatorname{Im} R^{A}(s)\right)=\frac{1}{2} \pi \alpha_{s}\left\langle\mathcal{O}_{\chi \mathrm{SB}}\right\rangle .
\end{aligned}
$$

One can obtain the left hand sides of these sum rules from the measured spectra determined from $\tau$ decays. The right hand side of [15a is given by the experimentally measured pion decay constant. It has been found [25] that both sides of (15a) agree very well. In the same way, the left hand side of (15b) has been determined. Here it has been found that the result agrees well with the conjecture that the four-quark condensate (14) factorizes into the square of the two-quark condensate [25]. This is exactly what large- $N_{c}$ considerations suggest. Below we will take the finding of [25] as a motivation to explore also the in-medium changes of the four-quark condensate (14) in the large- $N_{c}$ approximation. Note that we do not claim here that an arbitrary four-quark condensate is always connected to the square of the two-quark condensate. First of all, as we will see below, even for the four-quark condensate at hand, its in-medium change is not given by the change of the two-quark condensate (see also 24]). Second, from the point of view of chiral symmetry breaking, it makes sense to connect a chirally odd four-quark condensate, e.g. (14), to the 
two-quark condensate. Concerning chirally invariant four-quark condensates ${ }^{3}$, on the other hand, it is not so clear whether there should be any deeper connection to the two-quark condensate. In the present work we concentrate on one specific four-quark condensate, namely (14). In vacuum, this condensate seems to factorize [25].

From (15) it is obvious that both quantities $F_{\pi}^{2}$ and the four-quark condensate (14) can be regarded as order parameters of chiral symmetry breaking $(\chi \mathrm{SB})$. They must vanish if chiral symmetry is restored. To be precise, in a medium the relevant definition of $F_{\pi}$ is the coupling of the pion to the temporal component of the axial-vector current (10) [28]. Concerning their role as order parameters, we want to stress again that $F_{\pi}^{2}$ and $\alpha_{s}\left\langle\mathcal{O}_{\chi \mathrm{SB}}\right\rangle$ are actually more advantageous as compared to the two-quark condensate $\langle\bar{q} q\rangle$ which is the "standard" order parameter: The former are connected to quantities which are in principle measurable, namely the left hand sides of relations (15). ${ }^{4}$

The in-medium changes of $F_{\pi}^{2}$ are addressed in [29, 30] for a non-interacting pion gas (density $\rho_{\pi}$ ) and e.g. in [28] for a non-interacting (and cold) Fermi sphere of nucleons (density $\rho_{N}$ ). For finite temperatures (pion gas) one gets

$$
F_{\pi}^{2}(T)=F_{\pi}^{2}\left(1-\frac{4 \rho_{\pi}}{3 F_{\pi}^{2}}\right)
$$

with the (scalar) pion density

$$
\rho_{\pi}=3 \int \frac{d^{3} k}{(2 \pi)^{3} 2 E_{k}} \frac{1}{e^{E_{k} / T}-1} \stackrel{M_{\pi} \rightarrow 0}{\longrightarrow} \frac{1}{8} T^{2}
$$

and the pion energy $E_{k}=\sqrt{\vec{k}^{2}+M_{\pi}^{2}}$. For cold nuclear matter (nucleon Fermi sphere) the change of $F_{\pi}^{2}$ can be related to some low-energy constants of the chiral pion-nucleon Lagrangian. For details we refer to [28]. Numerically the result is

$$
F_{\pi}^{2}\left(\rho_{N}\right)=F_{\pi}^{2}\left(1-\frac{\rho_{N}}{\rho_{0}}(0.52 \pm 0.08)\right)
$$

where $\rho_{0}$ denotes nuclear saturation density. These results can be contrasted with the corresponding changes of the two-quark condensate $\langle\bar{u} u+\bar{d} d\rangle[29,31]$ :

$$
\frac{\langle\bar{u} u+\bar{d} d\rangle_{\text {pionic med. }}}{\langle\bar{u} u+\bar{d} d\rangle_{\mathrm{vac}}} \approx 1-\frac{\rho_{\pi}}{F_{\pi}^{2}}
$$

and 32

$$
\frac{\langle\bar{u} u+\bar{d} d\rangle_{\text {nucl. med. }}}{\langle\bar{u} u+\bar{d} d\rangle_{\mathrm{vac}}} \approx 1-\frac{\rho_{N} \sigma_{N}}{F_{\pi}^{2} m_{\pi}^{2}} \approx 1-\frac{1}{3} \frac{\rho_{N}}{\rho_{0}}
$$

The nucleon sigma term

$$
\sigma_{N}=m_{q} \frac{d m_{N}}{d m_{q}} \approx 45 \mathrm{MeV}
$$

which appears in (20) can be obtained within the framework of chiral perturbation theory from low-energy $\pi N$ scattering [33]. In principle, there are corrections to these linear-density results (16), (18), (19) and (20) which are of higher powers in the respective density. They involve correlations between the constituents of the medium. For the quark condensate in a pion gas this has been worked out in [31]. For all other cases model independent predictions are hard to make for the terms beyond linear order in the density.

Comparing (16) with (19) or (18) with (20) shows that a bold extrapolation of these formulae to the point where the respective order parameter vanishes would lead to different critical densities. This indicates a breakdown of the linear-density approximation for at least one of the compared respective quantities, probably for both.

Beyond these special cases of a (non-interacting) pion or nucleon gas the changes of $F_{\pi}^{2}$ are hard to estimate (except for specific models). The situation is somewhat better for the four-quark condensate (14) to which we turn for the rest of this work. We will compare the obtained results to the in-medium changes of the two-quark condensate $\langle\bar{u} u+\bar{d} d\rangle$. We note in passing that the in-medium behavior of $\langle\bar{s} s\rangle$ is different from the one of $\langle\bar{u} u+\bar{d} d\rangle$ [34]. Since we started out from the non-strange correlators $R^{V}$ and $R^{A}$, a comparison to $\langle\bar{u} u+\bar{d} d\rangle$ is most appropriate.

\footnotetext{
3 which appear e.g. in QCD sum rules for the $\omega$ meson [27]

${ }^{4}$ In practice this is a very tedious task: Access on $R^{V}$ can be obtained from dilepton spectra as discussed above; see also relation 9). Indeed, one tries to measure $R^{V}$ also in a medium (see e.g. [3]). In vacuum, $R^{A}$ is related to $\tau$ decays [16, [17] as already discussed. This is obviously difficult to measure in a medium.
} 


\section{RESONANCE GAS MODEL}

The in-medium changes described above in equations (16), (18), (19) and (20) have been determined under the assumption that the respective medium is described by a non-interacting gas of the respective most abundant particles (pions for a medium with finite temperature and nucleons for cold nuclear matter). Of course, to describe a medium with finite temperature and finite baryo-chemical potential one can easily generalize the previous results by using a gas of pions and nucleons (and anti-nucleons). ${ }^{5}$ Clearly, this is not the full story - except for low particle densities. Indeed, the in-medium expectation value (4) is defined with respect to all possible states. Beyond the consideration of single-particle states one has to take into account $n$-particle states with $n>1$, e.g. two-pion, pion-nucleon, nucleonnucleon, three-pion states and so on. The influence of $n$-particle states is accompanied by higher powers in the respective densities and therefore suppressed for low temperatures and low baryo-chemical potentials. This justifies the previous results as the respective leading terms in a low-density expansion. However, we want to understand now how to go beyond this linear-density approximation — at least approximately.

One might wonder why we only talked about pions and nucleons so far and not about their excitations. Indeed we will come to the excitations soon. From a principal point of view, however, we stress that there is no need to include them in the traces which appear in (4): For a complete set of states it is sufficient to consider all single- and many-body states built from the stable states (for flavor $S U(2)$ : pions, nucleons and anti-nucleons). As demonstrated long time ago in 35] the (unstable ${ }^{6}$ ) excitations emerge from the scattering phase shifts which come into play when considering many-body states in the traces in (4). E.g. a hadron resonance which is formed in pion-nucleon scattering is automatically taken into account when one considers the contributions of two-particle states (pion-nucleon) in the traces in (4).

On the other hand, it is in practice intractable to calculate the contributions of all $n$-body states to the expectation value (44). Now we can turn the previous argument around: Physically we expect that the contribution of a manybody state is the more important, the larger the many-body correlation is. Clearly, a large correlation is found, if a hadronic resonance is formed. Therefore one might approximate the complete set of (stable) many-body states by a sum over all one-body resonance states [36]. Below we will evaluate the in-medium two- and four-quark condensate in this resonance gas approximation (see also e.g. [31, 37, 38] for successful applications of that idea).

The following aspects should have become clear from the previous discussion:

a) The resonance gas approximation takes into account part of the many-body correlations of the stable states which form the medium. In that way one goes beyond the linear-density approximation discussed above.

b) The resonance gas approximation is not a systematic expansion in powers of the densities of (stable) medium constituents. One merely tries to take into account the most important parts of the higher density terms.

c) The resonance gas approximation can only make sense, if the many-body correlations are governed by hadron resonances. This excludes the application to systems with low temperatures and large baryo-chemical potential. In such systems nucleon-nucleon correlations are the most important ones.

In the resonance gas approximation we find

$$
\langle\mathcal{O}\rangle_{\text {med. }} \approx\langle 0|\mathcal{O}| 0\rangle+\sum_{X} \rho_{X}\langle X|\mathcal{O}| X\rangle
$$

where $|0\rangle$ denotes the vacuum state and $\mathcal{O}$ an arbitrary operator. $\rho_{X}$ is the scalar density of states $X$ :

$$
\rho_{X}=\int \frac{d^{3} k}{(2 \pi)^{3}} \frac{m_{X}}{E_{X}} n_{F / B}\left(E_{X}-s \mu\right)
$$

with

$$
n_{F / B}(E)=\left[\exp \left(\frac{E}{T}\right) \pm 1\right]^{-1}
$$

$E_{X}=\sqrt{m_{X}^{2}+\vec{k}^{2}}$ and $s=1$ for baryons, $s=-1$ for antibaryons and $s=0$ for mesons. The \pm sign and the label $F / B$ refer to baryons and mesons, respectively. We sum over all states $X$ identified by the particle data group [1].

\footnotetext{
${ }^{5}$ For our qualitative discussion we restrict ourselves to flavor $S U(2)$. For our quantitative analysis we will include strangeness.

${ }^{6}$ with respect to the strong interaction
} 
The normalization of the state $|X\rangle$ is chosen to be

$$
\left\langle X(\vec{k}) \mid X\left(\vec{k}^{\prime}\right)\right\rangle=\frac{E_{X}}{m_{X}}(2 \pi)^{3} \delta\left(\vec{k}-\vec{k}^{\prime}\right) .
$$

We already note here that this normalization is appropriate for heavy states since it has a proper non-relativistic limit. On the other hand, it is not quite adequate for Goldstone bosons where we would like to have states with a properly defined chiral limit. Of course, one can choose a different normalization, if one changes the definition of the density (23) accordingly. We will come back to that point below.

We have to discuss the evaluation of (22) separately for the two- and four-quark condensates. Concerning the type of hadron $X$ we also will distinguish in the following between Goldstone bosons and other hadrons. If $X$ is a Goldstone boson, the expectation values $\langle X|\bar{u} u+\bar{d} d| X\rangle$ and $\left\langle X\left|\mathcal{O}_{\chi \mathrm{SB}}\right| X\right\rangle$ can be calculated using current algebra [14, 39]. We postpone this discussion to section IV]

To calculate the contribution of non-Goldstone states to the in-medium part of the two-quark condensate we follow the approach of [31] and generalize it to finite baryo-chemical potential (see also [40, 41]). We approximate ${ }^{7}$

$$
\langle X|\bar{u} u+\bar{d} d| X\rangle \approx\left\langle X\left|u^{\dagger} u+d^{\dagger} d\right| X\right\rangle= \begin{cases}3-N_{s} & \text { for baryons, } \\ 2-N_{s} \text { for mesons }\end{cases}
$$

where $N_{s}$ denotes the number of strange quarks in the state $X$. For the sigma term [33] this amounts to the approximation

$$
\sigma_{X}= \begin{cases}\left(3-N_{s}\right) m_{q} & \text { for baryons } \\ \left(2-N_{s}\right) m_{q} & \text { for mesons }\end{cases}
$$

For the nucleon and the $\Delta(1232)$ we can get an idea how good this approximation is: Using $m_{q} \approx 7 \mathrm{MeV}$ and (21) we find that (27) underestimates $\sigma_{N}$ by about a factor of 2. On the other hand, a recent determination of $\sigma_{\Delta}$ yields $20.6 \mathrm{MeV}$ [2] in good agreement with (27). We will explore the uncertainty induced by the estimate (26) by an additional calculation where we simply increase $\sigma_{X}$ by a factor of 2 for each particle species. The in-medium twoquark condensate (but not the four-quark condensate) has also been calculated within a resonance gas approximation in [40]. There an estimate different from (26) has been used for the quark condensate within a resonance. We will come back to this point below.

Next we have to evaluate the expectation values appearing in (22) for the four-quark condensate (14). For the Goldstone bosons we note again that this can be performed using current algebra as will be discussed below in section IV] For all other hadrons it is difficult to get an estimate for $\left\langle X\left|\mathcal{O}_{\chi \mathrm{SB}}\right| X\right\rangle$. As already noted after equation (14) it is even a problem to pin down the vacuum expectation values of four-quark operators. For $\left\langle 0\left|\mathcal{O}_{\chi \mathrm{SB}}\right| 0\right\rangle$ it has been shown in [25] that it factorizes in the vacuum. In line with that finding, we use in the following the large- $N_{c}$ expansion [43, 44] where $N_{c}$ denotes the number of colors. For the operator of interest, $\mathcal{O}_{\chi \mathrm{SB}}$, we obtain [24, 45]

$$
\begin{gathered}
\left\langle 0\left|\mathcal{O}_{\chi \mathrm{SB}}\right| 0\right\rangle=O\left(N_{c}^{2}\right)=8\langle 0|\bar{u} u| 0\rangle^{2}+o\left(N_{c}\right) ; \\
\left\langle X\left|\mathcal{O}_{\chi \mathrm{SB}}\right| X\right\rangle=O\left(N_{c}^{2}\right)=8\langle 0|\bar{u} u| 0\rangle\langle X|\bar{u} u+\bar{d} d| X\rangle+o\left(N_{c}\right),
\end{gathered}
$$

if $X$ is a baryon;

$$
\left\langle X\left|\mathcal{O}_{\chi \mathrm{SB}}\right| X\right\rangle=o\left(N_{c}\right)
$$

if $X$ is a meson.

Therefore, it seems that to leading order in $1 / N_{c}$ we only must consider baryonic states and we can relate the in-medium four-quark condensate to two-quark condensates. However, there is an additional implicit $N_{c}$-dependence in $\rho_{X}$ which enters (22). We have shifted the discussion of that issue to appendix $\mathrm{A}$ The outcome of this discussion is that one should keep the respective leading order in $1 / N_{c}$ for every type of hadron to get a serious estimate for all regions of $T$ and $\mu$ (as long as $T$ is not too small, cf. point c) in the discussion above).

To obtain the respective leading $1 / N_{c}$ contribution for a specific type of hadron we need a closer look at the derivation of (29) and (30). We will summarize the essential points of [24] and apply it to our case of interest. First

\footnotetext{
${ }^{7}$ Note that this relation becomes so simple using the normalization (25).
} 
we note that the proof for (29) and (30) can be given for four-quark condensates involving white (color singlet) quarkantiquark operators. On the other hand, our condensate of interest (14) involves color octet operators. However, we can use a Fierz transformation to obtain

$$
\begin{aligned}
& \left\langle\left(\bar{u} \gamma_{\mu} \gamma_{5} \lambda_{a} u-\bar{d} \gamma_{\mu} \gamma_{5} \lambda_{a} d\right)^{2}-\left(\bar{u} \gamma_{\mu} \lambda_{a} u-\bar{d} \gamma_{\mu} \lambda_{a} d\right)^{2}\right\rangle= \\
& 2\left\langle(\underbrace{\bar{u} u+\bar{d} d}_{\sim f_{0}})^{2}\right\rangle+2\left\langle(\underbrace{\bar{u} i \gamma_{5} u-\bar{d} i \gamma_{5} d}_{\sim \pi^{0}})^{2}\right\rangle-8\langle\underbrace{\bar{u} i \gamma_{5} d}_{\sim \pi^{-}} \bar{d} i \gamma_{5} u\rangle+2\left\langle(\underbrace{\bar{u} i \gamma_{5} u+\bar{d} i \gamma_{5} d}_{\sim \eta, \eta^{\prime}})^{2}\right\rangle+2\left\langle(\underbrace{\bar{u} u-\bar{d} d}_{\sim a_{0}^{0}})^{2}\right\rangle-8\langle\underbrace{\bar{u} d}_{\sim a_{0}^{-}} \bar{d} u\rangle \\
& -\frac{2}{N_{c}}\left\langle(\underbrace{\left(\bar{u} \gamma_{\mu} \gamma_{5} u-\bar{d} \gamma_{\mu} \gamma_{5} d\right.}_{\sim \pi^{0}, a_{1}^{0}})^{2}-(\underbrace{\bar{u} \gamma_{\mu} u-\bar{d} \gamma_{\mu} d}_{\sim \rho^{0}})^{2}\right\rangle .
\end{aligned}
$$

For an easier discussion and for orientation we have attributed meson states to the respective operators which have the same quantum numbers. The four-quark operators which appear on the right hand side of (31) all have the generic structure $\bar{q} \Gamma q \bar{q} \Gamma^{\prime} q$ where $\Gamma$ and $\Gamma^{\prime}$ denote spinor and flavor, but no color structure. It has been shown in [24] that for this type of four-quark operators the following holds for expectation values with respect to single-particle states $X$ :

$$
\begin{aligned}
\left\langle X\left|\bar{q} \Gamma q \bar{q} \Gamma^{\prime} q\right| X\right\rangle= & \langle X|\bar{q} \Gamma q| X\rangle\left\langle 0\left|\bar{q} \Gamma^{\prime} q\right| 0\right\rangle+\langle 0|\bar{q} \Gamma q| 0\rangle\left\langle X\left|\bar{q} \Gamma^{\prime} q\right| X\right\rangle \\
& +\langle X|\bar{q} \Gamma q| 0\rangle\left\langle 0\left|\bar{q} \Gamma^{\prime} q\right| X\right\rangle+\langle 0|\bar{q} \Gamma q| X\rangle\left\langle X\left|\bar{q} \Gamma^{\prime} q\right| 0\right\rangle+\text { terms subleading in } 1 / N_{c}
\end{aligned}
$$

Obviously, the first two terms on the right hand side of (32) can contribute for every type of $X$, but only if $\Gamma$ and $\Gamma^{\prime}$ have the quantum numbers of the vacuum. This is only fulfilled by the first term appearing on the right hand side of (31). We can use again (26) to evaluate the respective contribution. This is the factorized part of the four-quark condensate.

Next we turn to the third and fourth term given on the right hand side of (32). These terms are contributions beyond factorization. First we note that these terms do not exist, if $X$ is a baryon: A quark-antiquark operator $\bar{q} \Gamma q$ cannot create a baryon $X$ from the vacuum. This simple fact finally leads to (29). If $X$ is a meson, the third and fourth term given on the right hand side of (32) only exist, if the quantum numbers of the meson match to the ones of $\bar{q} \Gamma q$ and $\bar{q} \Gamma^{\prime} q$. If this is the case, we need to know the overlap of the meson $X$ with the quark currents $\bar{q} \Gamma q$ and $\bar{q} \Gamma^{\prime} q$. In principle, such a question can be addressed within lattice QCD. However, for higher excited hadrons this is a difficult task. In principle, we would need this information. In practice, however, we will find that we can safely neglect the respective contributions. To see how this comes about, let us first discuss which hadrons are of relevance here: In view of (31) we see that particles which yield contributions in leading $1 / N_{c}$ order on top of the factorized part have quantum numbers of a) Goldstone bosons, b) $f_{0}$ or c) $a_{0}$. We disregard the vector and axial-vector mesons here since their contributions are $1 / N_{c}$ suppressed in (31). Concerning the group a) we note that only the excited states are of concern here: Goldstone bosons will be fully taken into account in section IV There, no approximations, especially no large- $N_{c}$ expansion is used besides the current algebra technique. Nonetheless, we note that we have checked explicitly that the current algebra calculations are not in contradiction to the large- $N_{c}$ considerations used here. Still we have to be concerned with excitations of the Goldstone bosons and with $f_{0}$ and $a_{0}$ states. All these states have masses of about $1 \mathrm{GeV}$ or higher [1]. ${ }^{8}$ As we will show below (cf. figure 4) these higher excited meson states are irrelevant from a practical point of view: For temperatures below, say, $200 \mathrm{MeV}$ relevant contributions to the sum (22) over all resonances come from the low-lying mesons (pseudoscalar and vector nonet) and from baryons. Higher lying mesons are strongly Boltzmann suppressed and — in contrast to high lying baryons — do not have large degeneracy factors. It turns out that the sum of all baryon resonances - albeit also Boltzmann suppressed adds up to a non-negligible contribution whereas the sum of all higher lying mesons remains irrelevant. Therefore, in practice we do not need the third and fourth term on the right hand side of (32) - except for Goldstone bosons to which we turn in the next section. Finally we note that in the spirit of the large- $N_{c}$ expansion we also treat the $\eta^{\prime}$ as a Goldstone boson (cf. e.g. [48] and references therein).

\footnotetext{
${ }^{8}$ We disregard the $f_{0}(400-1200)$ which we consider as a loosely bound meson-meson molecule and not a genuine hadron resonance state [46, 47].
} 


\section{GOLDSTONE BOSONS}

For Goldstone bosons we can use current algebra to calculate the contribution $\langle X|\mathcal{O}| X\rangle$ in (22). First, however, we shall choose a normalization which is more appropriate for Goldstone bosons. Instead of (23) and (25) we use for Goldstone bosons 39]

$$
\rho_{G}=\int \frac{d^{3} k}{(2 \pi)^{3}} \frac{1}{2 E_{G}} n_{B}\left(E_{G}\right)
$$

and

$$
\left\langle G(\vec{k}) \mid G\left(\vec{k}^{\prime}\right)\right\rangle=2 E_{G}(2 \pi)^{3} \delta\left(\vec{k}-\vec{k}^{\prime}\right) .
$$

With this normalization (which has a well-defined chiral limit) we get using current algebra e.g. for pions [14, 39]:

$$
\left\langle\pi^{a}|\mathcal{O}| \pi^{b}\right\rangle=-\frac{1}{F_{\pi}^{2}}\left\langle 0\left|\left[Q_{5}^{a},\left[Q_{5}^{b}, \mathcal{O}\right]\right]\right| 0\right\rangle
$$

with

$$
Q_{5}^{a}=\frac{1}{2} \int d^{3} x \bar{q}(x) \gamma_{0} \gamma_{5} \tau^{a} q(x)
$$

and a Gell-Mann flavor matrix $\tau^{a}$.

Obviously this can be generalized to other Goldstone bosons as well. As already mentioned we treat the whole pseudoscalar nonet as Goldstone bosons in the spirit of the large- $N_{c}$ approximation. We use the decay constant corresponding to the type of particle in the denominator of (35). For the flavor singlet we use $\tau^{0}=\sqrt{2 / 3} \mathbb{1}$.

It turns out that $\eta$ and $\eta^{\prime}$ do not contribute at all to $\left\langle\mathcal{O}_{\chi \mathrm{SB}}\right\rangle_{\text {med. }}$. They do contribute to the two-quark condensate (see (38) and (39) below). Why there is no contribution to the four-quark condensate can be explicitly seen, if one does not use current algebra directly for (14), but applies it separately to all the terms appearing on the right hand side of (31), i.e. after Fierz transformation. If $X$ is an $\eta$ or $\eta^{\prime}$, the first operator on the right hand side of (31) contributes via the first two terms on the right hand side of (32). The fourth operator on the right hand side of (31) contributes via the third and fourth term on the right hand side of (32). We have checked explicitly that in total these contributions indeed cancel.

With relations like (35) all expectation values can be traced back to vacuum expectation values. For the four-quark operator $\mathcal{O}_{\chi \mathrm{SB}}$ this leads to similar four-quark operators which in part deviate by their flavor content. We assume flavor symmetry of the vacuum to relate all resulting vacuum four-quark condensates to $\left\langle 0\left|\mathcal{O}_{\chi \mathrm{SB}}\right| 0\right\rangle$. No factorization (28) is needed here.

With the same technique (35) the two-quark condensate $\langle\bar{u} u+\bar{d} d\rangle_{\text {med. }}$ is related to the vacuum two-quark condensates $\langle 0|\bar{u} u+\bar{d} d| 0\rangle$ and $\langle 0|\bar{s} s| 0\rangle$ (the latter appears using kaons instead of pions in (35)). Again we assume flavor symmetry of the vacuum: $\langle 0|\bar{s} s| 0\rangle \approx\langle 0|\bar{u} u+\bar{d} d| 0\rangle / 2$.

Finally we want to comment on another order parameter of chiral symmetry breaking, the mixed quark-gluon condensate $^{9}\left\langle\bar{q} \sigma_{\mu \nu} G^{\mu \nu} q\right\rangle$ with the gluon field strength $G^{\mu \nu}$. In 11] it has been found that in lattice QCD the temperature dependence of the two-quark and the mixed condensate are practically the same. It has been suggested in 11] that this finding points towards a universal behavior of the order parameters. With the techniques presented

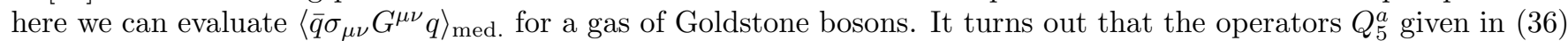
do not distinguish between $\mathbb{1}$, which appears in the two-quark condensate, and $\sigma_{\mu \nu} G^{\mu \nu}$, which appears in the mixed condensate. Therefore, for a gas of Goldstone bosons one gets

$$
\frac{\left\langle\bar{q} \sigma_{\mu \nu} G^{\mu \nu} q\right\rangle_{\text {med. }}}{\langle\bar{q} q\rangle_{\text {med. }}}=\frac{\left\langle 0\left|\bar{q} \sigma_{\mu \nu} G^{\mu \nu} q\right| 0\right\rangle}{\langle 0|\bar{q} q| 0\rangle} .
$$

On the other hand, for the four-quark condensate (14) the temperature dependence is different (see below). Therefore, it is questionable to conclude only from the temperature dependence of the two-quark and the mixed condensate that there is a universal behavior. Note that we do not claim that a gas of Goldstone bosons is equivalent to a lattice QCD calculation. We just have presented an example where the temperature dependence of two-quark and mixed condensate agrees, but where other order parameters show a different behavior.

\footnotetext{
${ }^{9}$ For the following comment we do not distinguish between flavor $S U(2)$ and flavor $S U(3)$.
} 


\section{RESULTS}

Now we can take all terms together. We find for the four-quark condensate:

$$
\begin{aligned}
\frac{\left\langle\mathcal{O}_{\chi \mathrm{SB}}\right\rangle_{\mathrm{med}} .}{\left\langle 0\left|\mathcal{O}_{\chi \mathrm{SB}}\right| 0\right\rangle}= & 1-\frac{2}{\pi^{2} F_{\pi}^{2}} \int_{0}^{\infty} d k \frac{k^{2}}{E_{\pi}} n_{B}\left(E_{\pi}\right)-\frac{1}{\pi^{2} F_{K}^{2}} \int_{0}^{\infty} d k \frac{k^{2}}{E_{K}} n_{B}\left(E_{K}\right) \\
& -\frac{m_{q}}{\pi^{2} F_{\pi}^{2} M_{\pi}^{2}} \sum_{B}\left(3-N_{s}\right) u m_{B} \int_{0}^{\infty} d k \frac{k^{2}}{E_{B}} n_{F}\left(E_{B}-s \mu\right) \\
& -\frac{m_{q}}{\pi^{2} F_{\pi}^{2} M_{\pi}^{2}} \sum_{M}\left(2-N_{s}\right) u m_{M} \int_{0}^{\infty} d k \frac{k^{2}}{E_{M}} n_{B}\left(E_{M}\right)
\end{aligned}
$$

where $M$ denotes meson resonances except for $\pi, K, \eta$ and $\eta^{\prime}$. The factor $u$ parameterizes the uncertainty connected to the estimate (26). In the figures below we will show calculations using $u=1$ in agreement with (26). In addition, we will use $u=2$ to explore the uncertainty of our estimate (cf. the discussion after (27)).

For the two-quark condensate we get

$$
\begin{aligned}
\frac{\langle\bar{u} u+\bar{d} d\rangle_{\text {med. }}}{\langle 0|\bar{u} u+\bar{d} d| 0\rangle}= & 1-\frac{3}{4 \pi^{2} F_{\pi}^{2}} \int_{0}^{\infty} d k \frac{k^{2}}{E_{\pi}} n_{B}\left(E_{\pi}\right)-\frac{1}{2 \pi^{2} F_{K}^{2}} \int_{0}^{\infty} d k \frac{k^{2}}{E_{K}} n_{B}\left(E_{K}\right) \\
& -\frac{1}{12 \pi^{2} F_{\eta}^{2}} \int_{0}^{\infty} d k \frac{k^{2}}{E_{\eta}} n_{B}\left(E_{\eta}\right)-\frac{1}{6 \pi^{2} F_{\eta^{\prime}}^{2}} \int_{0}^{\infty} d k \frac{k^{2}}{E_{\eta^{\prime}}} n_{B}\left(E_{\eta^{\prime}}\right) \\
& -\frac{m_{q}}{2 \pi^{2} F_{\pi}^{2} M_{\pi}^{2}} \sum_{B}\left(3-N_{s}\right) u m_{B} \int_{0}^{\infty} d k \frac{k^{2}}{E_{B}} n_{F}\left(E_{B}-s \mu\right) \\
& -\frac{m_{q}}{2 \pi^{2} F_{\pi}^{2} M_{\pi}^{2}} \sum_{M}\left(2-N_{s}\right) u m_{M} \int_{0}^{\infty} d k \frac{k^{2}}{E_{M}} n_{B}\left(E_{M}\right) .
\end{aligned}
$$

For the numerics we use the parameter values listed in table 【

\begin{tabular}{|c|c|c|}
\hline quantity & value in $\mathrm{MeV}$ & ref. \\
\hline \hline$F_{\pi}$ & 92.4 & {$[1]$} \\
\hline$M_{\pi}$ & 140. & {$[1]$} \\
\hline$m_{q}$ & 7. & {$[1]$} \\
\hline$F_{K}$ & 113. & {$[1]$} \\
\hline$F_{\eta}$ & 124. & {$[48]$} \\
\hline$F_{\eta^{\prime}}$ & 107. & {$[48]$} \\
\hline
\end{tabular}

TABLE I: Parameter values used for actual calculations.

As already noted we have treated the kaons as Goldstone bosons. Of course, one can study which formula we would have obtained, if we treated the kaon in the same way as the non-Goldstone bosons. This yields an opportunity to check our approximations. If kaons were considered as standard mesons instead of Goldstone bosons we observe first of all that there is indeed a relative factor 2 between the two kaon contributions in (38) and (39) - this is what one would expect from factorization: In (32) the first two terms on the right hand side give the leading $1 / N_{c}$ contribution, if $X$ is a kaon. The third and fourth term drop out since the quantum numbers of the operators appearing in (31) do not match to the kaon. Of course, we see this same relative factor 2 in all non-Goldstone contributions in (39) compared to (38). Next we compare the kaon contribution in (39) to the last contribution in (39): Instead of $1 /\left(2 \pi^{2} F_{K}^{2}\right)$ we would have for $u=1$ (the factor 4 comes from the four types of kaons)

$$
4 \frac{m_{q}}{2 \pi^{2} F_{\pi}^{2} M_{\pi}^{2}} M_{K}
$$


Indeed these two numbers deviate only by $6 \%$. This finding gives us some confidence that our approximations are not too bad.

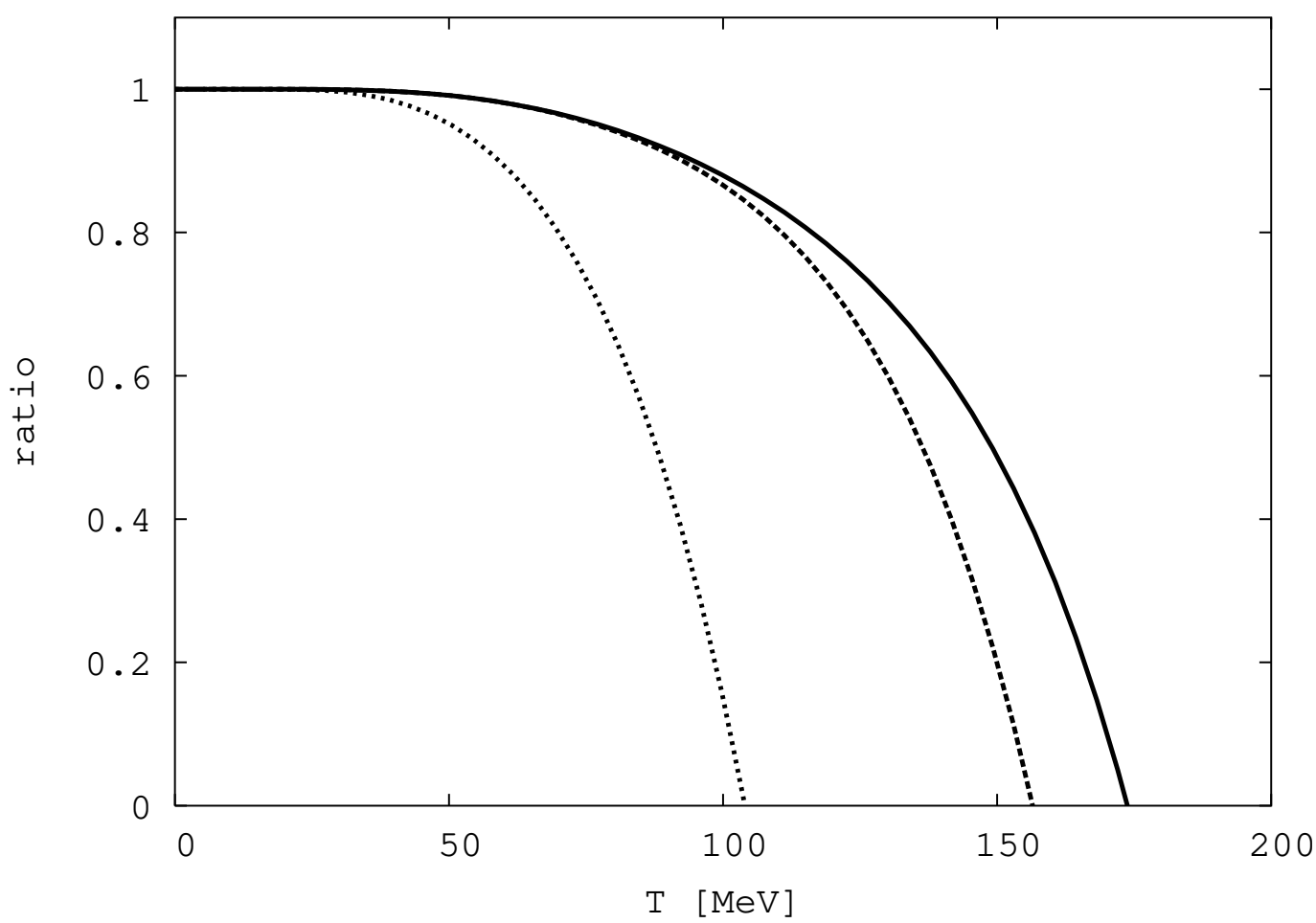

FIG. 1: Drop of the four-quark condensate [38) (for $u=1$ ) as a function of the temperature $T$ for three different baryo-chemical potentials: $\mu=0$ (full line), $\mu=400 \mathrm{MeV}$ (dashed), $\mu=800 \mathrm{MeV}$ (dotted).

With (38) at hand we can study how the four-quark condensate changes in a medium. This is depicted in figure 1 We see that the four-quark condensate drops both with temperature $T$ and baryo-chemical potential $\mu$. Of course, for the two-quark condensate there emerges a plot qualitatively similar to the one shown in figure 1. We will not display these results, but concentrate in the following on the line where the respective condensate vanishes. This is depicted in figure 2. From inspecting (38) and (39) one can already deduce that the four-quark condensate drops faster than the two-quark condensate and that the drop increases with the uncertainty parameter $u$. We observe these qualitative features also in figure 2] Quantitatively we deduce first of all that for vanishing chemical potential all lines yield a reasonable transition temperature between about 165 and $200 \mathrm{MeV}$. Without a bias which order parameter might be preferable we can view the lines in figure 2 as estimates for the transition line to a chirally restored state. The highest and the lowest line mark the uncertainty of the estimates. On the other hand, as discussed in great detail in section III we prefer the use of the four-quark condensate as a quantity which is closer connected to observables. As we will see next, the transition line extracted from the vanishing of the four-quark condensate shows an additional interesting feature.

An appealing aspect of the resonance gas approximation is the fact that one can easily calculate the energy and baryon density which correspond to given temperature and baryo-chemical potential:

$$
\begin{aligned}
\varepsilon & =\sum_{B} \int \frac{d^{3} k}{(2 \pi)^{3}} E_{B} n_{F}\left(E_{B}-s \mu\right)+\sum_{M} \int \frac{d^{3} k}{(2 \pi)^{3}} E_{M} n_{B}\left(E_{M}\right) \\
& =\frac{1}{2 \pi^{2}} \sum_{B, \text { no } \bar{B}} \int_{0}^{\infty} d k k^{2} E_{B}\left[n_{F}\left(E_{B}-\mu\right)+n_{F}\left(E_{B}+\mu\right)\right]+\frac{1}{2 \pi^{2}} \sum_{M} \int_{0}^{\infty} d k k^{2} E_{M} n_{B}\left(E_{M}\right)
\end{aligned}
$$

and

$$
\rho=\frac{1}{2 \pi^{2}} \sum_{B, \operatorname{no} \bar{B}} \int_{0}^{\infty} d k k^{2}\left[n_{F}\left(E_{B}-\mu\right)-n_{F}\left(E_{B}+\mu\right)\right] .
$$




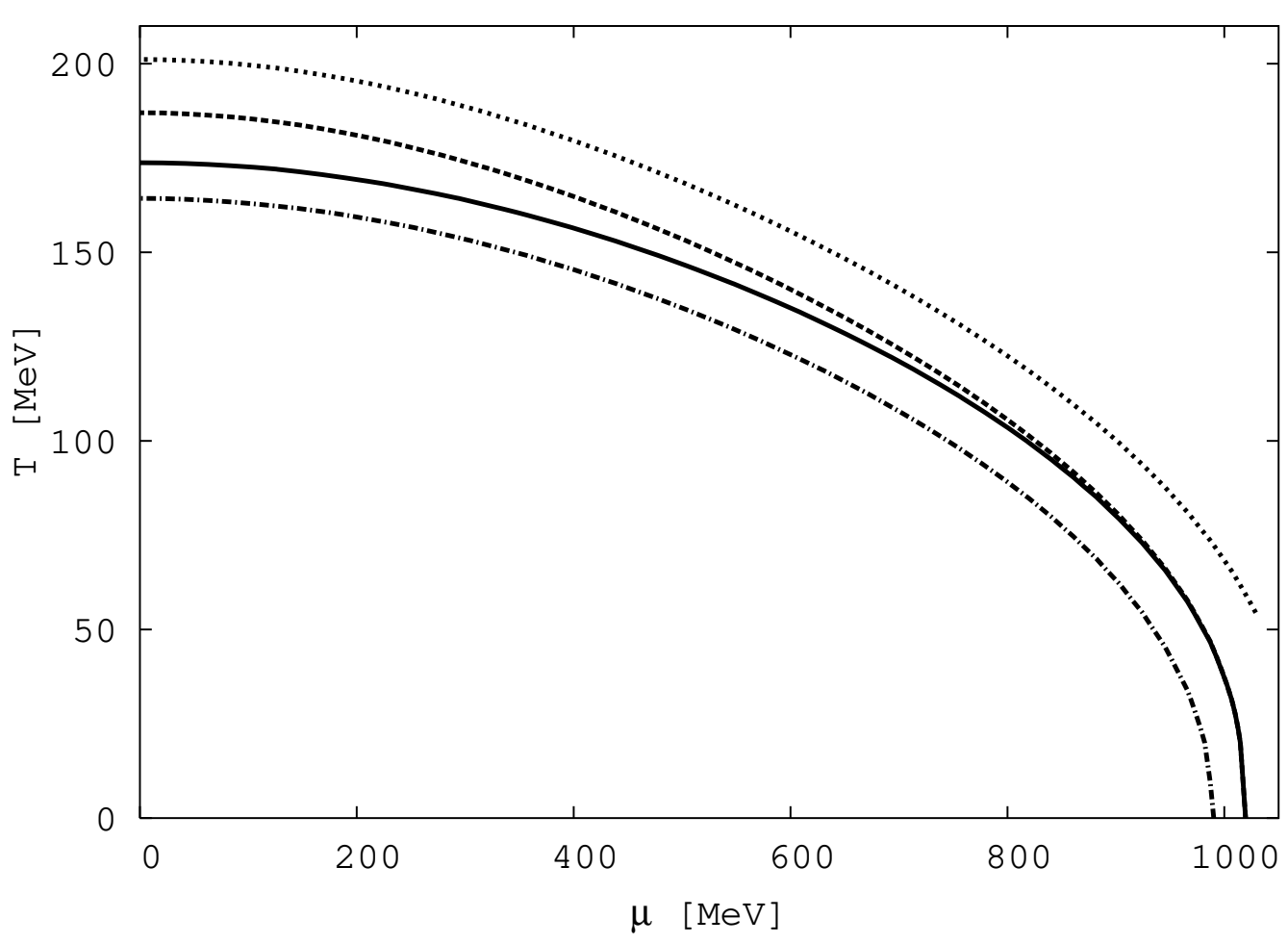

FIG. 2: Vanishing of two- and four-quark condensates in temperature-potential plane for different values of $u$. Lines enumerated from top to bottom. Line 1: Vanishing of two-quark condensate (39) with $u=1$. Line 2: Vanishing of two-quark condensate with $u=2$. Line 3: Vanishing of four-quark condensate (38) with $u=1$. Line 4: Vanishing of four-quark condensate with $u=2$.

Note that here $M$ denotes all mesons, including the Goldstone bosons.

Now we can study the vanishing of the condensates also as functions of the energy and baryon density. Figure 3 shows the corresponding results. We observe first of all that the critical energy density widely differs for the four different lines. This is not surprising: Differences in the temperature multiply to a high power for the determination of the corresponding energy density (for massless states we would have $\epsilon \sim T^{4}$ ). Figure 3 reveals a particularly interesting aspect: The energy density is extremely constant along the line where the four-quark condensate vanishes. Only for large baryon densities a different behavior sets in. This is the region of low temperatures (numerically $T<100 \mathrm{MeV}$ ) where we distrust the resonance gas approximation anyway. The critical energy density obtained from the vanishing of the four-quark condensate for $u=1$ is about $0.8 \mathrm{GeV} / \mathrm{fm}^{3}$. This agrees very well with lattice QCD results [37]. Note that the critical energy density obtained from the four-quark condensate for $u=2$ is not quite that constant - albeit still more stable than the critical lines obtained from the two-quark condensate. We recall that $u=1$ corresponds to our genuine estimate (26) whereas $u=2$ has been introduced by hand to get an idea about the uncertainties. We conclude that the four-quark condensate seems to be a very useful order parameter to determine the line of chiral symmetry restoration - at least within the resonance gas approximation.

Finally we shall discuss the uncertainties induced by the fact that not all resonances listed by the particle data group [1] are well established: We have checked that it makes no difference, whether we use all baryons of [1] or only the 3- and 4-star baryons. Figure 4 illustrates that it also makes no difference, whether one uses all mesons or only the lowest pseudoscalar and vector meson nonets. Using only the lowest octet and decuplet baryons instead of all baryons makes a difference, especially in an intermediate range of chemical potentials (see figure 4 ). We conclude that high-mass baryons are to some extent important due to their large multiplicities whereas high-mass mesons are not relevant. We recall that this is a fortunate situation, since we do not know the overlap of high-mass mesons with the corresponding quark currents which appears in (32). 


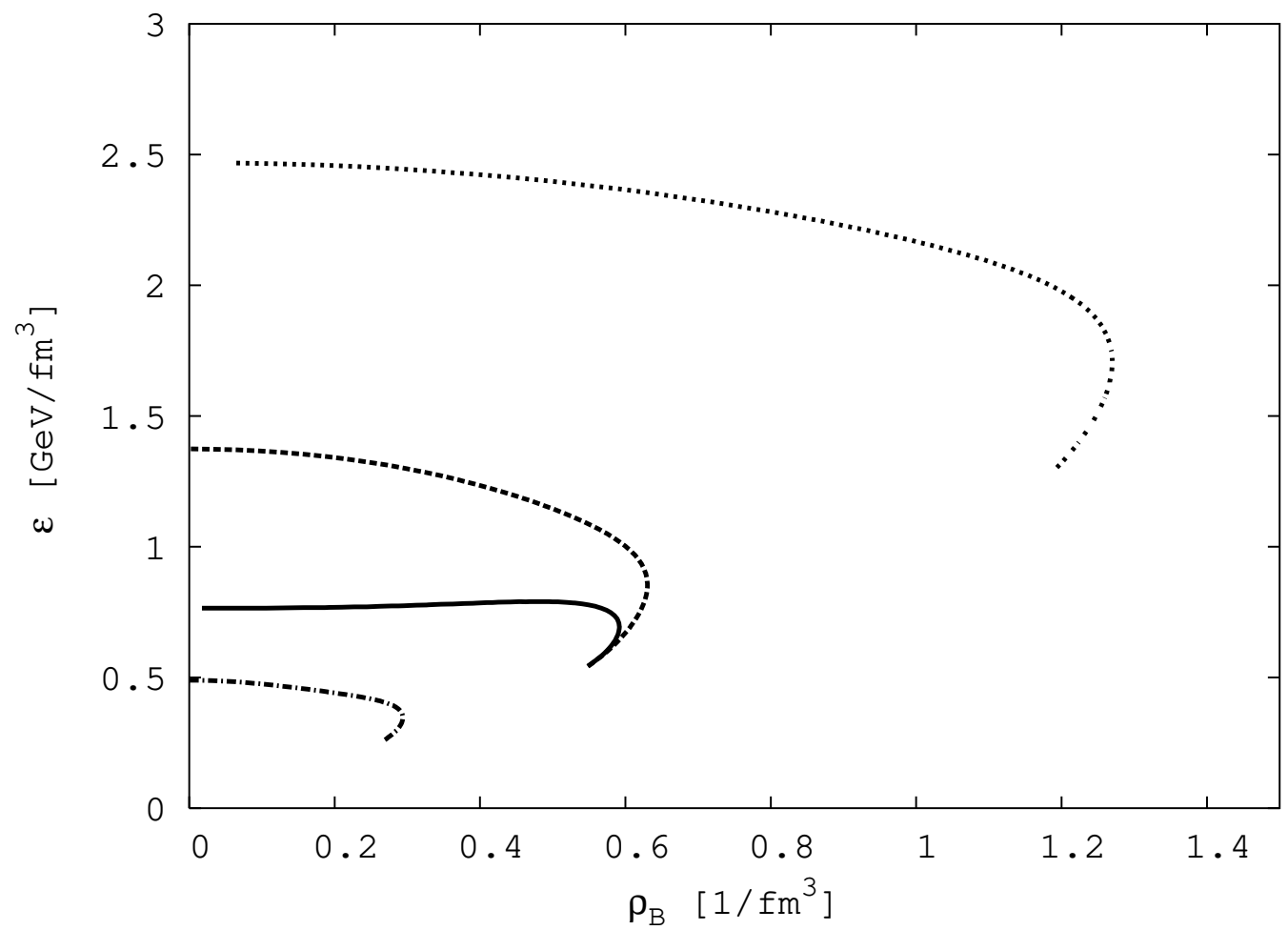

FIG. 3: Vanishing of two- and four-quark condensates in the plane of energy and baryon density for different values of $u$. Same line code as in figure 2

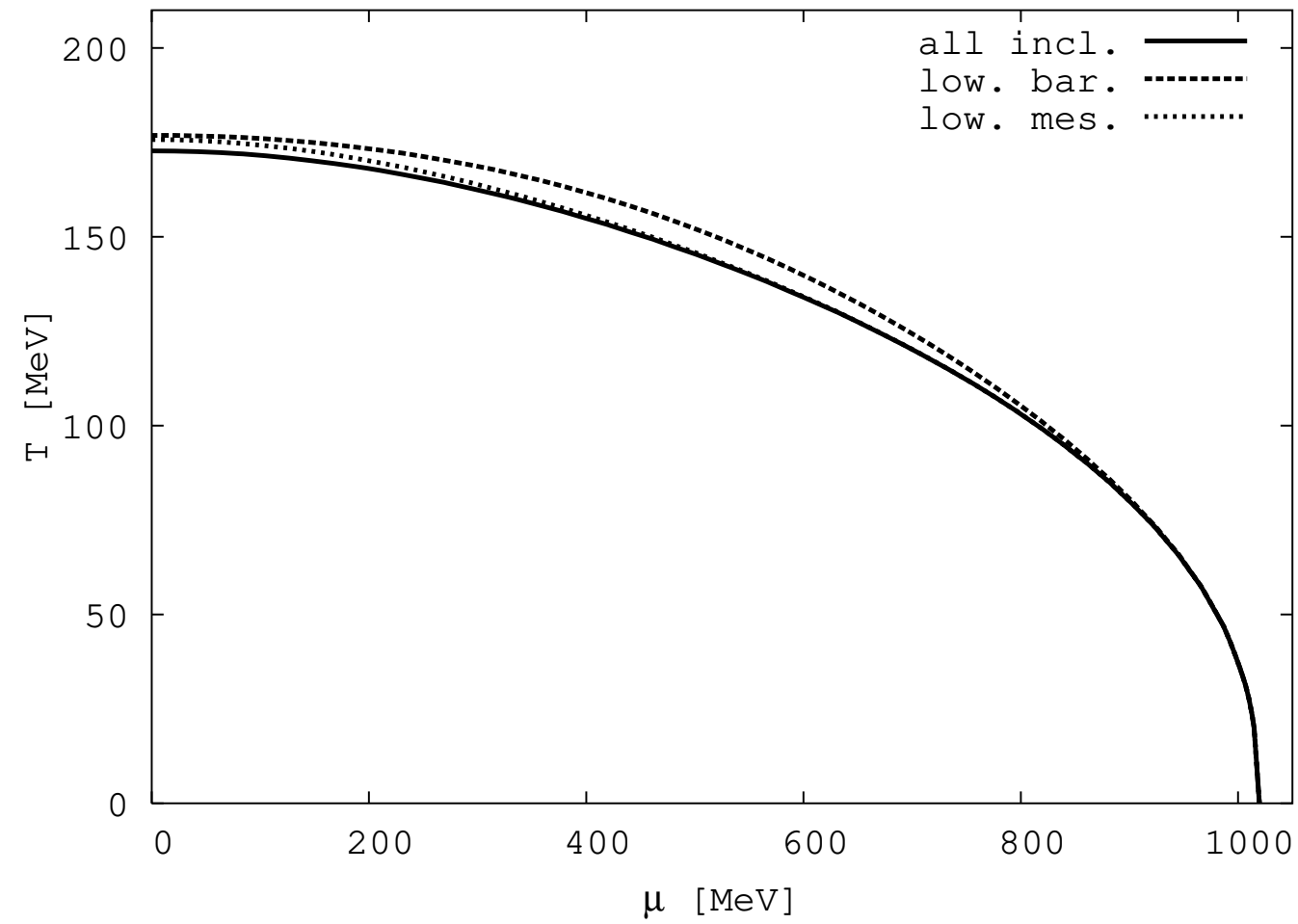

FIG. 4: Vanishing of the four-quark condensate (for $u=1$ ) for different sets of resonances. Full line: all mesons, all baryons; dotted line: lowest two meson nonets, all baryons; dashed line: all mesons, lowest two baryon multiplets. 


\section{SUMMARY AND OUTLOOK}

The present work consists of two parts: First, a motivation why the four-quark condensate (14) should be regarded as a useful order parameter of chiral symmetry restoration. Second, a calculation within the resonance gas approximation to determine how this four-quark condensate drops as a function of temperature and baryo-chemical potential and in particular where it vanishes. As our favorite prediction for the line of chiral symmetry restoration we regard the full lines in figures 2 and 3 . This yields a temperature $T_{c} \approx 174 \mathrm{MeV}$ at vanishing chemical potential and an energy density $\epsilon_{c} \approx 0.8 \mathrm{GeV} / \mathrm{fm}^{3}$ which stays remarkably constant as a function of baryon density or baryo-chemical potential.

The corresponding line which one obtains from the vanishing of the two-quark instead of the four-quark condensate does not show the same stability for the energy density $\epsilon_{c}$. This seems to be in contrast to the work [40] where also the in-medium change of the two-quark condensate has been determined within a resonance gas approximation. Here, we have to point out that our estimate for the resonance sigma terms (27) adopted from [31] differs from the estimate utilized in [40]. In the latter work the approximation [41]

$$
\sigma_{X} \approx M_{\pi}^{2} \frac{A}{m_{X}}
$$

with $0.9 \leq A \leq 1.2$ has been used for all resonances, no matter whether these are baryons or mesons. Such an approach does not agree with large- $N_{c}$ counting rules [24]: For baryons the sigma term should scale with $N_{c}$, whereas for mesons it should be constant. This requirement is satisfied by our approach: The factor $3-N_{s}$ in (26) and (27) is actually $N_{c}-N_{s}$ since a baryon consists of $N_{c}$ quarks [4]. In contrast, in the approximation (43) the sigma terms of baryons scale with $1 / N_{c}$ instead of $N_{c}$ since baryon masses - which appear in the denominator of (43) - scale with $N_{c}$. Note that the numerator in (43) cannot scale with $N_{c}$. Otherwise the scaling law for the meson resonances would be violated. Due to that deficiency we prefer our estimate (27).

Next we would like to discuss our approach in a more general context: In principle, we do not expect that any of our order parameters completely vanishes for high densities/temperatures. Only if chiral symmetry was an exact symmetry of QCD, the order parameters would exactly vanish in the chirally restored phase. In reality with finite current quark masses we expect a sizable drop of the order parameters at the phase transition or crossover point. After this transition point the order parameter presumably levels off. We have sketched the expected behavior in figure 5. Clearly the exact behavior of the order parameters near the transition point is hard to extract from low-density expansions or more general from an approach which uses only the degrees of freedom which are relevant below the transition point. Therefore we should not expect to obtain a completely accurate description of the transition region. Nonetheless, if one has a model which still works reasonably well in the region where the drastic change of the order parameter sets in, the estimated transition point should not deviate too much from the real one. Such a scenario is sketched in figure 5

For the evaluation of the in-medium behavior of the four-quark condensate we have used two crucial approximations: First, a resonance gas to model the in-medium system, and second, large- $N_{c}$ arguments to evaluate the four-quark condensate for the non-Goldstone bosons. Concerning the latter approximation it is unfortunately hard to check its validity for the real world of $N_{c}=3$. For general arguments in favor of the large- $N_{c}$ approximation we refer to [3, 44] and to the large amount of works which cite these seminal papers.

Concerning the resonance gas approximation there is at least one aspect which might lead to additional nonnegligible contributions beyond the ones obtained from the resonance gas: Since the pions are very light, the threshold for their interactions $\left(2 M_{\pi}\right)$ is not significantly Boltzmann suppressed for the temperatures we are interested in (close to $200 \mathrm{MeV})$. On the other hand, if all the pion-pion interactions were mediated by narrow resonances, the relevant scale would not be set by the threshold but by the resonance masses. Indeed, there are arguments in favor of a resonance saturation of the low-energy constants which determine the pion-pion interaction [8, 49, 50]. However, these resonances, in particular the $\rho$-meson, are not narrow. Therefore a significant interaction already shows up at energies below the $\rho$-meson mass. Hence, pion-pion interactions should be taken into account beyond the resonance gas approximation. For the two-quark condensate at finite temperature and vanishing baryo-chemical potential these interactions have been evaluated up to three loops within chiral perturbation theory in [31]. It turned out that the effects induced by these interactions are not completely negligible, but small as compared to the combined effects from the non-interacting pions, nucleons and the resonances [31]. To clarify whether this also is true for the four-quark condensate is devoted to future work. Another possible and straightforward extension of our approach is the inclusion of isospin and strangeness chemical potential. Also this is beyond the scope of the present work. 


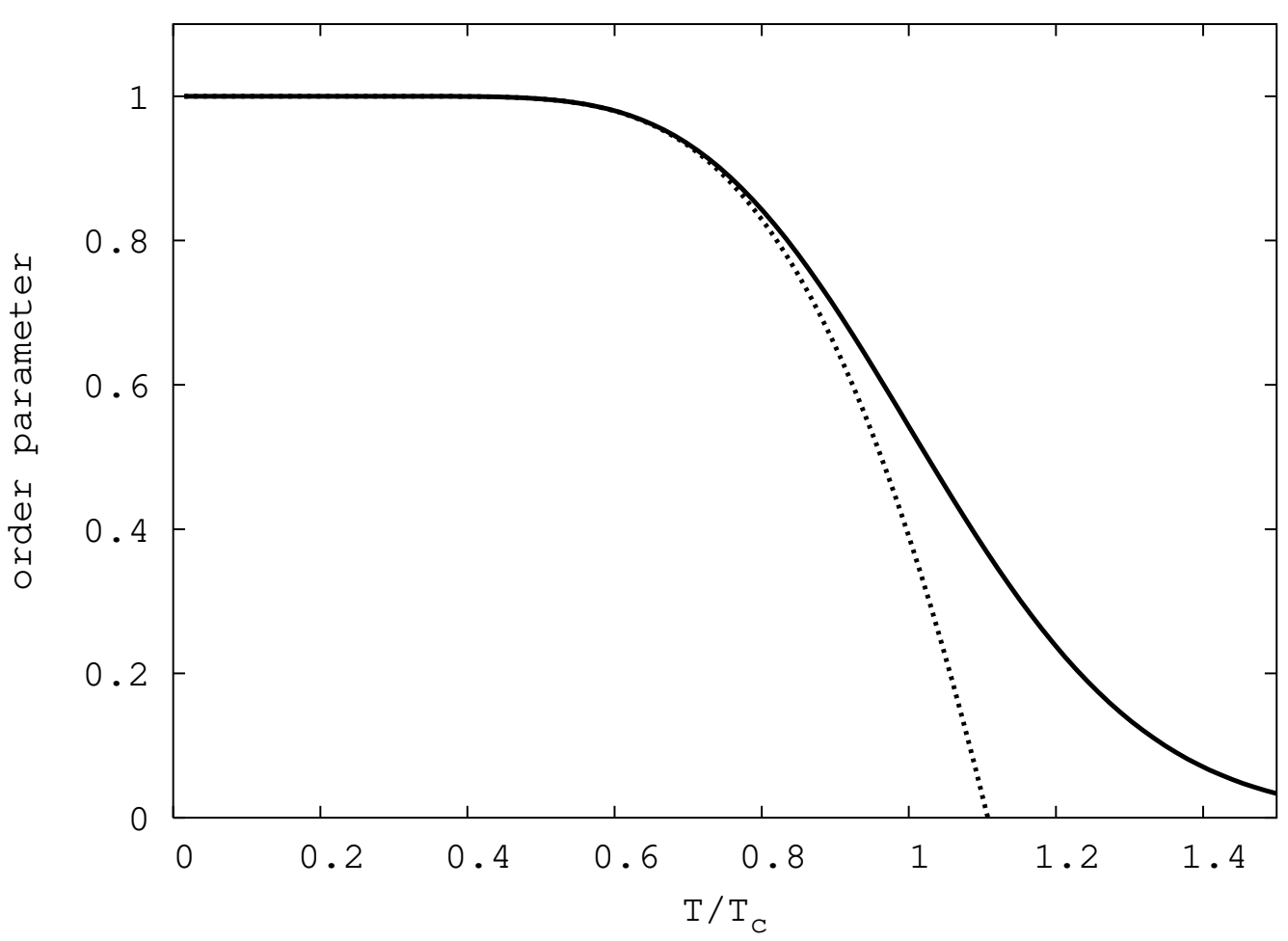

FIG. 5: Sketch of the real behavior of an order parameter as a function of temperature (full line) and of a reasonable approximation obtained from the low-density degrees of freedom (dotted line).

\section{Acknowledgments}

S.L. acknowledges the support of the European Community-Research Infrastructure Activity under the FP6 "Structuring the European Research Area" programme (HadronPhysics, contract number RII3-CT-2004-506078).

\section{APPENDIX A: RESONANCE DENSITY AND LARGE NUMBER OF COLORS}

From (29) and (30) it seems that to leading order in $1 / N_{c}$ we only must consider baryonic states. However, there is an additional implicit $N_{c}$-dependence in $\rho_{X}$ as we will discuss now. We will find that we have to distinguish different regimes for the baryo-chemical potential. This is intimately connected to the fact that the masses of baryons scale with $N_{c}$ [44]:

$$
m_{X}=O\left(N_{c}\right),
$$

if $X$ is a baryon, whereas

$$
m_{X}=O\left(N_{c}^{0}\right),
$$

if $X$ is a meson. The reason is that a baryon consists of $N_{c}$ quarks while a meson is a quark-antiquark state.

We start with the case that the chemical potential is small compared to all baryon masses. For temperatures which are reasonable for hadronic degrees of freedom we have for baryons

$$
\sqrt{m_{X}^{2}+\vec{k}^{2}}-\mu>m_{X}-\mu \gg T .
$$

In this case we can replace the Fermi distribution in (23) by a Boltzmann distribution and we can treat the problem 
non-relativistically. This yields for baryons

$$
\begin{aligned}
\rho_{X} & \approx \int \frac{d^{3} k}{(2 \pi)^{3}} \frac{m_{X}}{E_{k}} \exp \left(-\frac{E_{k}-\mu}{T}\right) \approx \exp \left(-\frac{m_{X}-\mu}{T}\right) \int \frac{d^{3} k}{(2 \pi)^{3}} \exp \left(-\frac{\vec{k}^{2}}{2 m_{X} T}\right) \sim \exp \left(-\frac{m_{X}-\mu}{T}\right)\left(m_{X} T\right)^{3 / 2} \\
& =o\left(N_{c}^{3 / 2} \exp \left(-N_{c}\right)\right) .
\end{aligned}
$$

For mesons there is no $N_{c}$-dependence, $\rho_{X}=O\left(N_{c}^{0}\right)$. Since the expectation value of the four-quark condensate is enhanced by $N_{c}$ for baryons (cf. (29) and (30), we find in total that the relative importance of a baryonic contribution as compared to a mesonic one is $o\left(N_{c}^{5 / 2} \exp \left(-N_{c}\right)\right)$. In the large- $N_{c}$ limit an exponential suppression overwhelms any power enhancement. Therefore, for small baryo-chemical potentials the in-medium change of the four-quark condensate is dominated by mesons.

The situation changes, however, for larger baryo-chemical potentials to which we turn next: For $m_{X}-\mu=O\left(N_{c}^{0}\right)$ there is no implicit $N_{c}$-dependence left in $\rho_{X}$, i.e. $\rho_{X}=O\left(N_{c}^{0}\right)$ even for baryons. ${ }^{10}$ In this case, the in-medium four-quark condensate is dominated by baryons.

To get a serious estimate for all regions we should keep the respective leading order in $1 / N_{c}$ for every type of hadron.

[1] S. Eidelman et al. (Particle Data Group), Phys. Lett. B592, 1 (2004).

[2] S. Scherer, Adv. Nucl. Phys. 27, 277 (2003), hep-ph/0210398.

[3] R. Rapp and J. Wambach, Adv. Nucl. Phys. 25, 1 (2000), hep-ph/9909229.

[4] M. Creutz, Quarks, Gluons and Lattices, Cambridge Monographs On Mathematical Physics (Cambridge University Press, Cambridge, UK, 1983).

[5] C. T. H. Davies et al. (HPQCD), Phys. Rev. Lett. 92, 022001 (2004), hep-lat/0304004.

[6] F. Karsch, Lect. Notes Phys. 583, 209 (2002), hep-lat/0106019.

[7] M. C. Birse, Phys. Rev. C53, 2048 (1996), hep-ph/9602266.

[8] J. Gasser and H. Leutwyler, Ann. Phys. 158, 142 (1984).

[9] M. Knecht, B. Moussallam, J. Stern, and N. H. Fuchs, Nucl. Phys. B471, 445 (1996), hep-ph/9512404.

[10] P. Pascual and R. Tarrach, QCD: Renormalization for the Practitioner, vol. 194 of Lect. Notes Phys. (Springer, Berlin, 1984).

[11] T. Doi, N. Ishii, M. Oka, and H. Suganuma, Phys. Rev. D70, 034510 (2004), hep-lat/0402005.

[12] Z. Fodor and S. D. Katz, JHEP 03, 014 (2002), hep-lat/0106002.

[13] C. R. Allton et al., Phys. Rev. D66, 074507 (2002), hep-lat/0204010.

[14] T. Hatsuda, Y. Koike, and S. H. Lee, Nucl. Phys. B394, 221 (1993).

[15] C. Gale and J. I. Kapusta, Nucl. Phys. B357, 65 (1991).

[16] K. Ackerstaff et al. (OPAL), Eur. Phys. J. C7, 571 (1999), hep-ex/9808019.

[17] R. Barate et al. (ALEPH), Eur. Phys. J. C4, 409 (1998).

[18] S. Weinberg, Phys. Rev. Lett. 18, 507 (1967).

[19] M. E. Peskin and D. V. Schroeder, An Introduction to Quantum Field Theory (Perseus, Cambridge, Massachusetts, 1995).

[20] M. A. Shifman, A. I. Vainshtein, and V. I. Zakharov, Nucl. Phys. B147, 385, 448 (1979).

[21] J. I. Kapusta and E. V. Shuryak, Phys. Rev. D49, 4694 (1994), hep-ph/9312245.

[22] D. T. Barfoot and D. J. Broadhurst, Phys. Lett. B166, 347 (1986).

[23] C. A. Dominguez and K. Schilcher, Phys. Lett. B581, 193 (2004), hep-ph/0309285.

[24] S. Leupold, Phys. Lett. B616, 203 (2005), hep-ph/0502061.

[25] J. Bordes, C. A. Dominguez, J. Penarrocha, and K. Schilcher, JHEP 02, 037 (2006), hep-ph/0511293.

[26] V. A. Novikov, M. A. Shifman, A. I. Vainshtein, M. B. Voloshin, and V. I. Zakharov, Nucl. Phys. B237, 525 (1984).

[27] B. Steinmüller and S. Leupold (2006), hep-ph/0604054.

[28] U.-G. Meißner, J. A. Oller, and A. Wirzba, Annals Phys. 297, 27 (2002), nucl-th/0109026.

[29] J. Gasser and H. Leutwyler, Phys. Lett. B184, 83 (1987).

[30] J. Gasser and H. Leutwyler, Nucl. Phys. B307, 763 (1988).

[31] P. Gerber and H. Leutwyler, Nucl. Phys. B321, 387 (1989).

[32] E. G. Drukarev and E. M. Levin, Prog. Part. Nucl. Phys. 27, 77 (1991).

[33] J. Gasser, H. Leutwyler, and M. E. Sainio, Phys. Lett. B253, 252 (1991).

[34] J. R. Pelaez, Phys. Rev. D66, 096007 (2002), hep-ph/0202265.

10 We note in passing that the contributions of antibaryons are always, i.e. for arbitrary positive baryo-chemical potential, suppressed by $N_{c}^{5 / 2} \exp \left(-N_{c}\right)$. 
[35] R. Dashen, S.-k. Ma, and H. J. Bernstein, Phys. Rev. 187, 345 (1969).

[36] R. Hagedorn, Nuovo Cim. Suppl. 3, 147 (1965).

[37] F. Karsch, K. Redlich, and A. Tawfik, Eur. Phys. J. C29, 549 (2003), hep-ph/0303108.

[38] F. Karsch, K. Redlich, and A. Tawfik, Phys. Lett. B571, 67 (2003), hep-ph/0306208.

[39] V. L. Eletsky, Phys. Lett. B299, 111 (1993).

[40] D. Toublan and J. B. Kogut, Phys. Lett. B605, 129 (2005), hep-ph/0409310.

[41] A. Tawfik and D. Toublan, Phys. Lett. B623, 48 (2005), hep-ph/0505152.

[42] V. Bernard, T. R. Hemmert, and U.-G. Meissner, Phys. Lett. B622, 141 (2005), hep-lat/0503022.

[43] G. 't Hooft, Nucl. Phys. B72, 461 (1974).

[44] E. Witten, Nucl. Phys. B160, 57 (1979).

[45] S. Leupold and M. Post, Nucl. Phys. A747, 425 (2005), nucl-th/0402048.

[46] G. Colangelo, J. Gasser, and H. Leutwyler, Nucl. Phys. B603, 125 (2001), hep-ph/0103088.

[47] J. R. Pelaez, Phys. Rev. Lett. 92, 102001 (2004), hep-ph/0309292.

[48] B. Borasoy, Eur. Phys. J. C34, 317 (2004), hep-ph/0402294.

[49] G. Ecker, J. Gasser, A. Pich, and E. de Rafael, Nucl. Phys. B321, 311 (1989).

[50] G. Ecker, J. Gasser, H. Leutwyler, A. Pich, and E. de Rafael, Phys. Lett. B223, 425 (1989). 